\title{
Responses of the splanchnic circulation to ischaemia
}

\author{
ADRIAN MARSTON \\ From The Middlesex and Royal Northern Hospitals, London
}

The distribution of the cardiac output is conventionally shared between six major circulatory components, that is, to the brain, heart, kidneys, bone and skeletal muscle, skin and viscera. This last comprises the splanchnic circulation, which arrives through the three great visceral arteries (coeliac axis, superior and inferior mesenteric artery) and is drained, via the liver, into the hepatic veins. The organs supplied include the liver, spleen and pancreas, stomach, duodenum and small and large intestine. In practice, the one area in which deprivation of blood supply carries important physiological consequences is the alimentary tract, because the other territories are irrigated by a double circulation. This chapter will, therefore, be mainly concerned with events in the mesenteric circulation.

\section{Structure}

\section{GROSS}

The coeliac axis, superior mesenteric artery (SMA) and inferior mesenteric artery (IMA), carry between them from a fifth to a quarter of the total cardiac output. Of the three arteries the most important is without doubt the superior mesenteric (SMA), because it is functionally an end artery, and its routes of collateral supply from above and below are precarious. At the upper and lower ends of the abdomen, there are connexions with extra-coelomic vessels which protect against the consequences of any fall in blood flow. Thus, if the coeliac axis is occluded the deficiency is quickly made up from diaphragmatic, intercostal and oesophageal vessels, supplemented by contributions from the abdominal wall, and in the same way, because the pelvic organs have a rich supply from the branches of the internal iliac artery, ischaemic problems in the lower bowel are a clinical rarity. Acute occlusion of the SMA, however, is badly tolerated. (fig1).

\section{MINUTE}

The final vessels springing from the arcades and penetrating the wall of the bowel run tangentially and penetrate alternative aspects of its circumference. Henceforward, the arrangement of the vasculature reflects the avidity of the various layers of the bowel

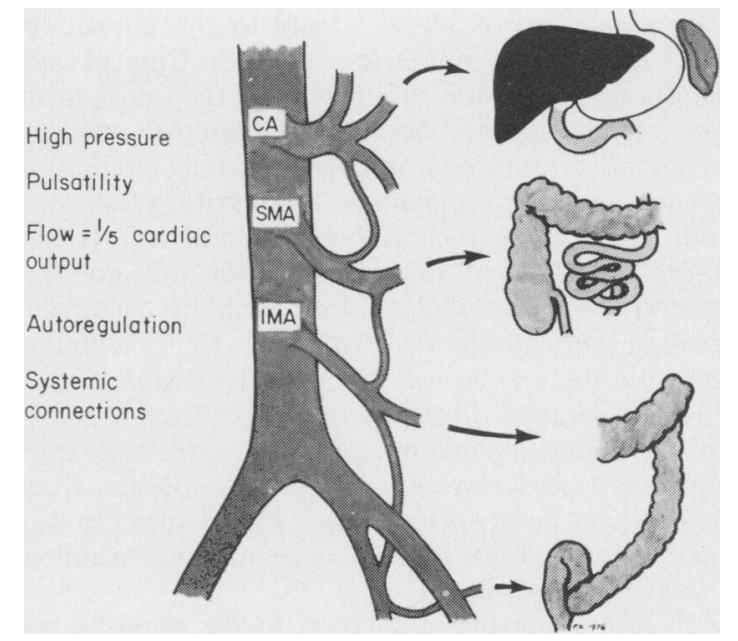

Fig 1 The main visceral arteries.

for oxygen. A few minor vessels run backwards to supply the serosa and outer longitudinal muscle coats (which are fairly undemanding structures) and from there a number of straight vessels, arranged in roughly rectangular fashion, penetrate to the submucosa, where they form a rich plexus which extends as a vascular carpet uninterruptedly from the duodenum to the anal canal, on which the mucosa rests. This provides a very efficient collateral network, containing both arteries and veins, but there seems to be but little direct communication between them. Arteriovenous anastomoses have been sought in the gastrointestinal tract for many years, and the classical work (Spanner, 1932) suggested that they were abundant. However, studies using microdissection techniques (Barclay and Bentley, 1949), thick film sections (Boulter and Parks, 1960), and labelled microspheres, have been unable to confirm the presence of such shunts in any significant number in the small bowel, which receives the major proportion of the intestinal blood supply, although there may be some in the stomach and colon.

From the submucosal plexus spring the arterioles to the villi, which are the final determinant of metabolic exchange. A single central arteriole runs up the 
centre of the villus and arborizes at its tip into a dense capillary network, which communicates with the venules lying beneath the basement membrane. The position of the artery in the stroma, and the vein in the subepithelium, is of crucial importance to intestinal function, and will be discussed in detail below.

\section{Physiology}

The regulation of blood supply to the alimentary tract has received rather less attention from physiologists than have the circulations to the brain, heart and kidney, perhaps because the extremely efficient collateral arrangement in the splanchnic circulation renders vascular accidents in this territory less common than are myocardial or cerebral infarctions. From the point of view of evolution, the gut is a much older organ, and its blood supply is capable of dealing with all sorts of extremes. To the running and hunting animal it is a low priority area, and in circumstances of threat, such as flight from a predator or following a major wound, the alimentary tract can safely be deprived of blood. Nonetheless, there is a critical point, below which intestinal infarction takes place and this process, once initiated, is almost impossible to stop.

In examining the circulation to the intestine, we need to consider both total and regional intestinat flow.

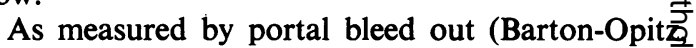
1911), isotope clearances (Svanik, 1973; Lundgren: 1974) and electromagnetic flowmeters (Burns and Schenck, 1967, 1969), the mesenteric circulation of the healthy dog receives some 20 per cent of the cardiac output and if these results are extrapolated t $\overline{8}$ man (and there is evidence that this is valid) th 8 amounts to a figure of some 1200 to $1800 \mathrm{ml}$ per miry that is, under resting conditions. It would seem likely from the evolutionary point of view that the figure would fall during exercise and rise during digestion and most of the evidence supports this (Wade et a.9. 1956; Vatner et al, 1970). The means by which 'd 8 . gestive hyperaemia' is brought about are complexz and include the action of vasodilator hormones such as secretin, cholecystokinin, glucagon, GIP and" VIP (Lundgren, 1974), accumulation of metabolites i the gut wall (Lundgren, 1974; Marston, 1977), and the operation of locally induced mucosal reflexes possibly through the action of 5HT receptors (Lund gren, 1974).

What matters, however, is not so much the tot quantity of blood which reaches the alimentar tract, but what happens to it once it arrives. We ows our knowledge of the distribution of blood fow throughout the layers of the gut wall to the work of Professor Bjorn Folkow and his school in Götebơr|⿳亠丷厂犬

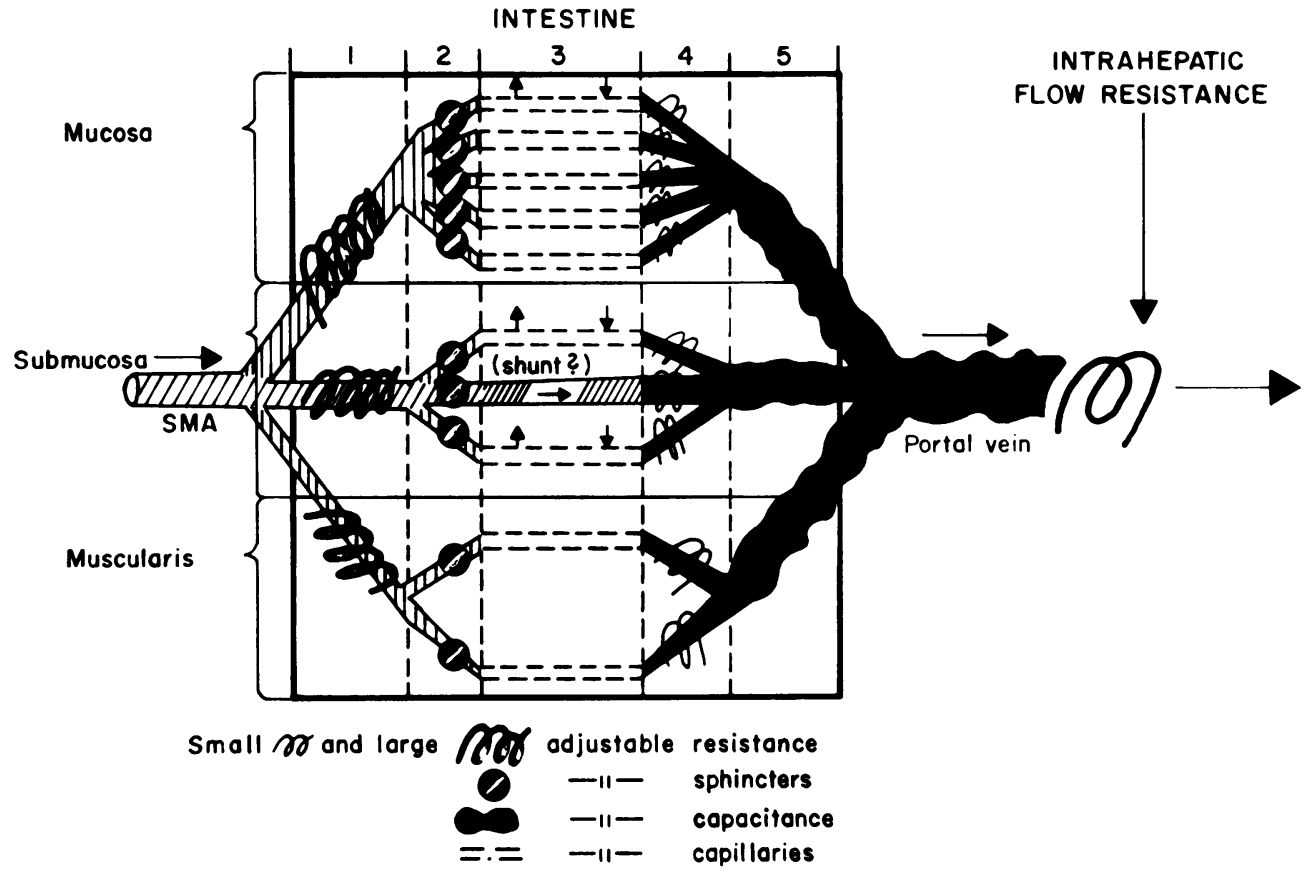

Fig 2 Flow and pressure in the gut wall. (By courtesy of Professor B Folkow and the Editor, Gastroenterology.) 
It is generally true that blood flow to an organ is directly related to its demands for oxygen. The smooth muscle of the gut is a relatively inactive structure, and its oxygen consumption, and hence blood flow, is low. The needs of the submucosa and mucosa are somewhat higher, because of their increased level of cellular turnover, but their oxygen consumption is out of all proportion to this, and approximates to three times the basic metabolic demand. This is because the intestinal capillary is concerned not only with the nutrition of its target tissues, but also with the absorption and secretion of material in and out of the lumen of the gut, which amounts to a large multiple of the blood volume, during the course of 24 hours. The inner layers of the gut thus have a much higher blood flow than their oxygen requirements alone would warrant, and there exists, furthermore, a system of internal switching mechanisms which redistributes flow from one layer to another, in response to altering circumstances, even while total blood flow remains constant (Lundgren, 1974) (fig 2).

Although the capillaries form a crucial area, in that the secretion of mucus and enzymes and the absorption of nutriments takes place across their membranes, they themselves play little or no part in the regulation of flow, but rather accept what they are given. The number and distribution of open capillaries is regulated by the pre-capillary sphincters, and the total amount of blood available is determined by the arterioles, which constitute the main resistance. The factors influencing the diameter of the intestinal arteriole are illustrated in figure 3.

The most useful studies of distribution of blood flow throughout the layers of the gut have been carried out using lipid soluble gases such as $85 \mathrm{Kr}$, whose clearance is flow-dependent (Boley et al, 1969; Biber et al, 1973; Lundgren, 1974; Marston, 1977). Study of the washout curves of such an injected isotope demonstrate characteristic clearance rates in separate zones of the intestinal wall, corresponding to the cell layers. Most of the blood goes to the mucosa, which has a flow rate about three times that of the muscle, but there exists in the deep parts of the crypts and in the submucosa, a region of exceptionally high vascularity, which carries a flow of some 400 to $600 \mathrm{ml}$ per minute per $100 \mathrm{~g}$ of tissue, contrasted with overall average of 30 to $35 \mathrm{ml}$ per minute per $100 \mathrm{~g}$ of tissue. When the vessels are artificially dilated with an agent such as isopropyl noradrenaline, this deep submucosal layer now takes an even higher proportional volume of blood. It is possible that bacterial endotoxin operates in the same way.

The existence of a high-demand, high-flow zone in the submucosa is not altogether surprising. This is an area of intense secretory activity. Also, it is here that
FACTORS WHICH REDUCE DIAMETER

$\begin{array}{ll}\text { MECHANICAL } & \begin{array}{l}\text { Elostic Recoil } \\ \text { Musculor 'Squeeze' } \\ \text { Abdominal Pressure }\end{array} \\ \text { NERVOUS } & \text { Sympothetic (Short acting) } \\ \text { HUMORAL } & \begin{array}{l}\text { Adrenaline, Norodrenaline } \\ \text { ADH, Angiotensin } I, C o .\end{array}\end{array}$

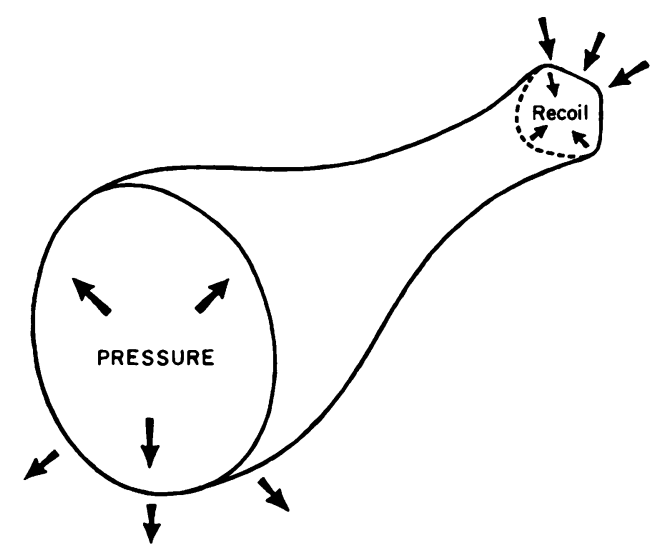

FACTORS WHICH INCREASE DIAMETER

MECHANICAL Hydrostatic Pressure

NERVOUS Sympothetic (long acting)

HUMORAL $\quad \mathrm{CO}_{2}$ Loctate. Krebs cycle intermediotes $\mathrm{Mg}$.

Fig 3 Factors influencing the diameter of the intestinal arteriole.

the new epithelium is manufactured, as the cells move upwards along the basement membrane to be shed at the tips of the villi. An additional explanation for the apparent presence of high flow in this area is the location of the intestinal counter-current exchange system.

Physiologists have for a long time been concerned with the presence of arteriovenous oxygen shunting through the intestinal circulation, as represented by a component of the isotope clearance curve, which clears more rapidly than could be explained by passage through the entire capillary network. The most obvious basis for this would be the existence of anatomical AV connexions, but, as has already been mentioned, these do not appear to exist. Evidence is accumulating that the 'hairpin' arrangement of vessels in the villus, so reminiscent of what is seen in the nephron, in fact represents a counter-current exchange mechanism, which helps to maintain the integrity of the serum electrolytes and proteins in the face of tidal fluxes in osmolarity of the gut contents. Absorption (particularly of water) is delayed, and at the same time this arrangement (see fig 4) ensures 


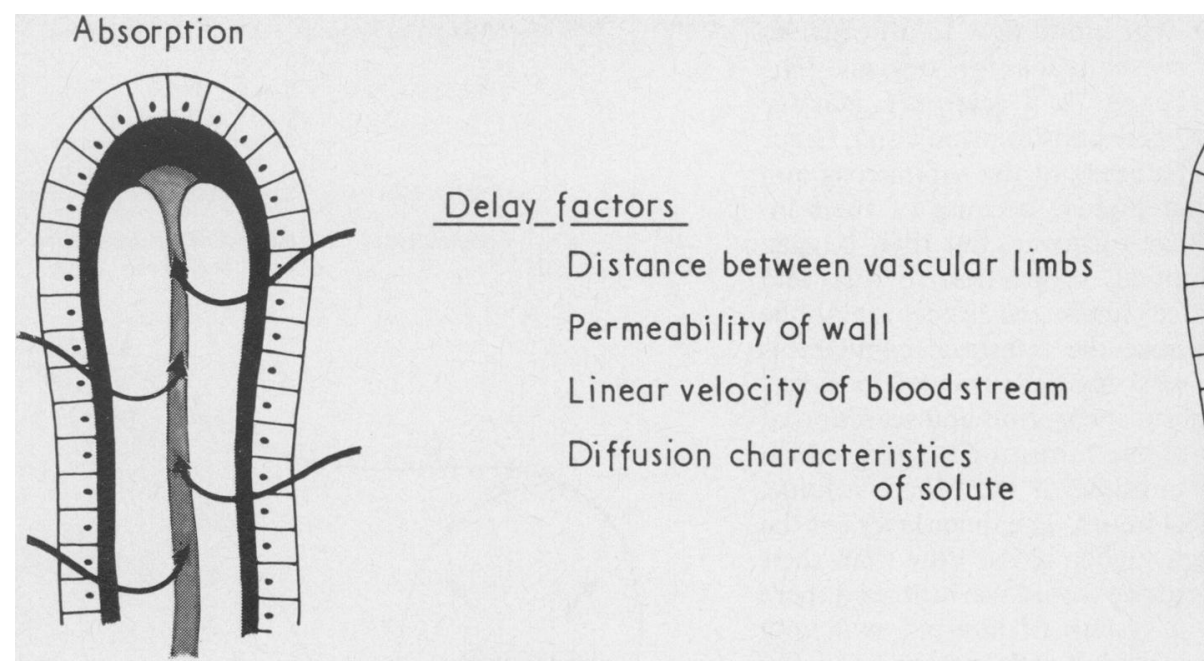

Fig 4 The counter-current exchange mechanism in the villous circulation.

that a solute in the bloodstream will not be lost into the gut, because of the existence of an arteriovenous concentration gradient at the base of the villus. Oxygen can diffuse across the two vascular endothelia in the same way. The counter-current exchange becomes progressively more efficient as velocity is reduced up and down the limbs of the loop, so that low-flow states (as may result from a fall in input pressure or an increase in blood viscosity) will lead to shunting of oxygen across the base of the villus, thus effectually devascularizing the tip. Such a process may result either in luminal ulceration or in the separation of a pseudomembrane, though what actually determines which of these results accrues, remains unknown.

\section{Pathophysiology: Causes and effects of reduced intestinal blood flow}

\section{CAUSES}

There is an equilibrium between the various factors which increase or reduce blood supply to the intestinal mucosa, and if any one of these is disturbed, the balance between the potentially invasive bacteria within the lumen and the mucosal defences will be upset, with resulting destruction of the intestinal wall (fig 5). In particular, the pressure within the lumen, which has a direct effect on diameter and on radial tension, is crucial, and as the gut dilates there is a progressive fall off in intramural blood flow (Boley et al, 1969).

The more important causes of acute reduction in blood flow are: mesenteric embolus; acute hypotension associated with visceral artery stenosis; digitalis toxicity; small vessel disease (arteritis); obstruction clostridial infection.

Mesenteric embolus is now a rarity, but the cond tion is of great scientific interest because it is the exact clinical parallel of the standard laboratosy model of intestinal ischaemia, namely, the occlugion of the blood supply to the midgut loop of a heafth animal with normal vessels. Most patients who des from intestinal infarction do not, in fact, hake emboli. Some of them have atheromatous lesions at the origins of their visceral arteries, but one-third of fatal cases of gut necrosis show no such abnormalit:. Moreover, stenoses, plaques and even complete occlusions of the mesenteric vessels are frequent found at necropsy in individuals who have had alimentary symptoms during life. For this reasow, 'mesenteric thrombosis' can no longer be considered as a cause of acute intestinal ischaemia, because the relationship between the vascular lesion and what occurs in the gut is so uncertain, especially in its timing. There is plenty of circumstantial evidence, however, that persons who have atheromatous plaques on the visceral arteries risk sustaining intestinal infarction if for some reason the pressure across this lesion is reduced (Marston, 1963). Any drop in central arterial pressure will of course lead to an increase in sympathetic drive and in the level of circulating catecholamines, which (at first, at any rate) results in further vasoconstriction in the meser teric circulatory bed.

An additional cause of intestinal ischaemia, $\mathbb{\$}$ seen in clinical practice, is the use of cardiac glycio sides. Digitalis in moderate dosage increases intestinal blood flow through its effect on the output of 


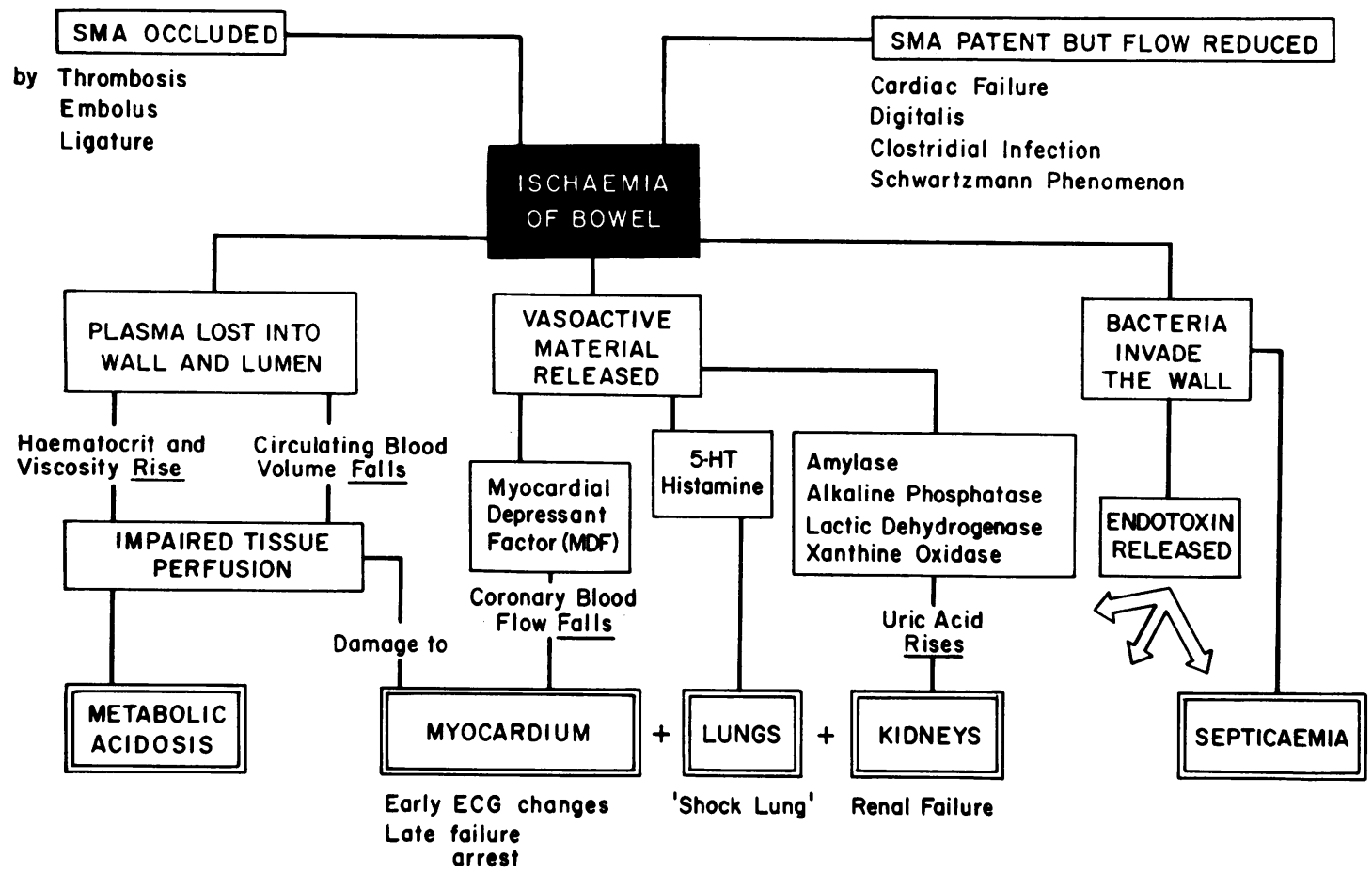

Fig 5 The physiological disturbance following acute intestinal ischaemia.

the failing heart, but it nonetheless is a powerful vasoconstrictor to the gut vessels, and there are many reports in the literature of cases of total midgut necrosis resulting from overdosage of digitalis (Polansky et al, 1964; Pawlik and Jacobson, 1974).

Inflammatory lesions of the vessels in the gut wall complicate many immune-complex diseases such as polyarteritis, lupus erythematosus, rheumatoid disease and the macroglobulinaemias. However, involvement of these vessels usually arises late in the course of the illness and is frequently a terminal event. In any case, these conditions are all rare, and the intestinal element is not of much clinical importance. Small vessel occlusion due to increased intraluminal pressure (in practice resulting from obstruction) is of much more immediate concern. As already mentioned, obstruction leads to a rise in intraluminal pressure with a concomitant fall in blood flow, and ischaemic mucosal changes are so frequently seen proximal to carcinoma or stricture. The so-called 'stercoral' ulceration is in fact localized ischaemic necrosis. Interestingly, the area immediately next to the growth, because it is tethered and unable to expand, often preserves its blood flow and remains normal, whereas a few centimetres proximal there may be quite severe damage (Saegesser et al, 1972).

The question arises as to whether, given a suffi- ciently strong challenge from the lumen, the intestine can undergo necrosis in the presence of a completely normal blood supply. There is some evidence that this can happen. Outbreaks of clostridial infection in north Germany (Zeissler and Rassfeld-Sternberg, 1949) and in New Guinea (Murrell et al, 1966) suggested that a bacterium equipped with a sufficiently powerful vasoconstrictor exotoxin can in fact bring about necrosis of the intestinal wall in a healthy young subject. However, the discovery of such organisms in a necrotic gut in an elderly person with arterial disease perhaps does not have the same significance. In such circumstances it is difficult to distinguish between causative and opportunistic infections.

\section{EFFECTS}

The locally generated effect on the bowel of deprivation of blood has effects on the organism as a whole. While they of course interact, it is easier to look at them separately.

\section{Effects on the bowel}

The most immediate effect of SMA occlusion as seen in the laboratory is an intense and violent spasm of the gut, resulting in the appearance of pale circular stripes as the muscle squeezes the blood into the veins. The arteries collapse and the veins distend. 
This initial period of hypercontractility is followed by relaxation and distension, and the accumulation of oedema fluid in the gut and the peritoneal cavity. After eight to 10 hours frank haemorrhage begins to occur and at the point of death the bowel from the duodeno-jejunal flexure to the transverse colon is dark red, without sheen, and soaked in bloody oedema. The serosal coat is raised in blebs, the muscular layers congested, and the mucosa grossly swollen with a massive secretion of serosanguinous fluid. The veins are engorged with blood, but usually not thrombosed.

There have recently been detailed studies on the early cellular changes resulting from ischaemia (Chiu et al, 1972). These are as follows:-

\section{Grade 0 Normal villi}

Grade 1 Development of a subepithelial space (Grunhagen's space) at the apex of the villus

Grade 2 Extension of the space with lifting of the epithelial layer from the lamina propria

Grade 3 Massive epithelial lifting along the sides of the villus, with denudation at the tips

Grade 4 Complete denudation of the villi with the lamina propria and dilated capillaries exposed

Grade 5 Digestion and disintegration of the lamina propria with haemorrhage and ulceration.

Electron microscope studies suggest that the fluid in the subepithelial space originates partly from the capillary but also from the lumen of the bowel. There is a direct correlation between the speed of development of mucosal damage and the fall of blood flow in the SMA, and with complete occlusion of the SMA grade 5 mucosal damage may appear within 30 minutes. As ischaemia progresses the tips of the villi begin to slough and a membrane of necrotic epithelium, fibrin, inflammatory cells and bacteria, accumulates. Later on oedema and haemorrhage occur in the submucosa, while there is a steady progression of necrotic change from the lumen outwards, until, in the worst affected area, no trace of mucosal detail remains. While these events occur in the mucosa, there is progressive emptying of the arterial tree with engorgement of the veins, some of which thrombose. There is evidence that the oedema fluid and the venous engorgement result from the effect of the portal pressure (which is well maintained) on the damaged intestinal capillary (see below (Marston, 1962, 1963). The initially brisk inflammatory response in the wall of the bowel, with accumulation of polymorphonuclear leucocytes, is gradually replaced by a diminution of inflammatory cell elements, presumably due to depletion of their numbers by anoxia, with no corresponding replacement from the arterial circulation. The bowel wall becomes progressively thinned, as the mucosa separates and sloughs into the lumen, and at nine to ten hours perforation may occur, but is by no means $\stackrel{0}{+}$ invariable.

\section{General effects}

Acute and total intestinal ischaemia, whatever theo cause, produces an immensely complex physiologicalo disturbance which is difficult to analyse because so $\frac{\overline{\bar{D}}}{\bar{D}}$ many of the factors influence each other in unpre- $\mathbb{\mathbb { Q }}$ dictable ways. Once the physiological changes have been initiated, it is extremely difficult to arrest the ${ }^{\mathrm{c}}$ process by any form of therapy, but at the same time. it is true both of the laboratory animal and of the $\vec{\overrightarrow{ }}$ patient with a mesenteric embolus that death ${ }_{\mathscr{N}}^{\omega}$ generally occurs before intestinal infarction is com-웅 plete, and furthermore (in the laboratory) that release ${ }_{\text {is }}$ of the occluded SMA after more than a few hours of

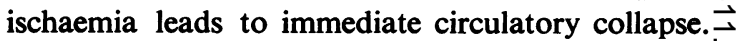
The two main components of the physiological-disturbance are, on the one hand, redistribution of 6 body fluid, and on the other absorption of vaso-음 active material from the ischaemic bowel. The rela-tive importance of these interacting factors has been $C$ the subject of a rather academic debate over the last 20 years.

\section{Redistribution of body fluid}

The infarcted intestine behaves very much as $\frac{8}{0} \mathrm{~V}$. massive skin burn, which is not surprising when ofe bears in mind the relative surface areas of these two epithelia. Occlusion of the SMA leads to progressive $\vec{\partial}$ plasma loss into the wall and lumen of the gut and into the peritoneal cavity, with a resulting haemo- $\frac{\mathbb{Q}}{2}$ concentration resulting in decreased tissue perfusion $\vec{\Rightarrow}$ all over the body, not least in the intestine itself.

In 1962 we demonstrated a mean fall in blood $\stackrel{\Im}{-}$ volume of 34 per cent between the time of SMAO occlusion and death in the experimental laboratory (Marston, 1963, 1964). The red cell mass during thiso time fell by 10 per cent, while the plasma volume fello by 54 per cent on average. The haematocrit fre quently reached a level of 65 to 70 per cent immedi-ô ately before death. This massive degree of haemoconcentration and fluid loss occurred without any? significant rise in portal pressure, so that the well $>$ known syndrome of 'hepatic outflow obstruction'? which tends to occur in dogs in response to stress, was ruled out as a cause. The conclusion reached on the basis of these studies was that the plasma leakage 0 was occurring from the venous side of the circula- $-\omega$ tion, into the wall and lumen of the bowel, via the damaged intestinal capillary.

These results have been confirmed since by Tjiong듇 et al (1974) who investigated the relative importance? of fluid shift and metabolic change during and after occlusion of the SMA in 11 splenectomized dogs $\frac{O}{D}$ 
Following measurements of blood volume and total body water the SMA was clamped, and the clamp released four hours later, following which the spaces were again measured, the measurement being repeated after a further two hours. At the same time, blood gases, serum electrolytes and osmolarity were estimated. These authors were able to confirm the major shifts in body fluids which we observed in our studies in 1962, and at the same time demonstrated that blood cultures, in spite of changes characteristic of endotoxin absorption, were negative. While blood volume, total body water, serum potassium, $\mathrm{pCO}_{2}$ and bicarbonate all declined, the arterial $\mathrm{pO}_{2}$ remained relatively constant.

\section{Absorption of vasoactive material}

At the same time as plasma loss is proceeding, chemical substances are absorbed from the ischaemically challenged intestine, which can be demonstrated either by examining the small physiological changes which occur in the intact animal (Williams et al, 1969; Lefer, 1970), or by investigating the pharmacological potential of the portal blood by its effect on standard biological preparations (Kobold and Thal, 1963). Systemic effects occur before any discernible histological damage, let alone gangrene, has occurred in the ischaemic bowel. Very brief periods of SMA occlusion lead to electrocardiographic changes and a decrease in cardiac output, and some authors have postulated the presence (Lefer, 1970) of a (hitherto unidentified) 'myocardial depressant factor' in very early intestinal ischaemia. Transitory rises in arterial pressure and in coronary, renal and cerebral blood flow occur, which are followed by progressive increases in systemic vascular resistances with a concomitant fall in tissue flow, at a stage where the gut remains intact (Marston, 1977).

As ischaemia progresses, these early and subtle changes are replaced by a gross physiological disturbance involving all systems and organs (fig 5). Massive amounts of vasoactive material are produced in the ischaemic bowel and absorbed by the peritoneum, veins and lymphatics. The portal blood can be demonstrated to contain increased concentrations of catecholamines, histamine, 5-hydroxytryptamine, ferritin, $\beta$-glucuronidase, transaminases and alkaline phosphatase. Additionally, the portal and later the systemic blood is hyperkalaemic, and has a low pH. Moreover, this is a situation which will result in very high concentrations of Gram-negative bacterial endotoxin, and many of the systemic effects of acute intestinal ischaemia are attributable to the presence of such substances.

\section{Manifestations of intestinal ischaemia}

In practical terms, we can distinguish three situations which are encountered by the clinician. These are:

\section{ACUTE INTESTINAL FAilure}

By this is meant a clinical state in which the metabolic needs of the small intestine outrun its blood supply, resulting in necrosis, threatened or complete, of the major part of the alimentary tract. The factors leading to this have been described above, as have the metabolic consequences. It is probable that many examples of 'haemorrhagic', ' necrotizing', 'pseudomembranous', etc, enterocolitis are, in fact, manifestations of this state of affairs (Marston, 1964, 1977).

2 CHRONIC INTESTINAL ISCHAEMIA

The concept of limitation of arterial supply to the intestine, resulting in ischaemic pain much as is felt in the myocardium or lower limbs, has great appeal to the surgeon, and up until a few years ago there were still many enthusiastic reports in the surgical literature of so-called 'cures' of obscure abdominal pain achieved by reconstruction of the visceral arteries (Morris et al, 1962; Reul et al, 1974). However, the situation is not at all simple, and there is reason to doubt whether in fact so-called 'intestinal angina' really exists as an important clinical entity. Stenosis and even complete occlusion of the main visceral trunks is a common necropsy finding in subjects who experienced no digestive disturbance during life, and the lesions may even be so gross as to limit resting flow. Patients with this sort of arteriographic abnormality have been investigated with great assiduity, and because such lesions are very easily reproduced in the animal laboratory, there is a thorough back up of laboratory experience. At the present time, however, no clinical or experimental worker has ever succeeded in demonstrating a structural or functional abnormality in the intestine which can be consistently correlated with diminution in blood supply, unless there is an infarction.

It seems logical to suppose that reduction in blood supply to the alimentary tract would produce absorptive or exsorptive abnormalities which could be measured in the clinical laboratory. In fact, studies of this nature are not easy to come by, and tend to disagree. Thus while some workers were unable to demonstrate any functional abnormality at all in patients with angiographic blocks of major visceral arteries others have noted steatorrhoea which disappeared following arterial reconstruction. One study demonstrated abnormal absorption of carbohydrate and fat, measured respectively by the dxylose and $\pm^{131}$ triolein techniques, which was corrected by aorto-SMA bypass. Others (Dardik et al; Delmont, 1971) report abnormal serum carotene levels and increases in faecal fat, or delayed absorption of radio-iodinated triolein, and impaired d- 
xylose absorption which reverted to normal following arterial reconstruction.

It seems to be an 'all-or-nothing' situation in that the intestine remains normal and uncomplaining until such point as its cells can no longer metabolize or resist bacterial challenge, at which time they disintegrate and produce appearances which the pathologist can recognize under his microscope.

\section{FOCAL LESIONS OF THE INTESTINE}

Infarction is a matter of degree. In the gut, a period of anoxia may be followed by total necrosis, a transient inflammatory response, or the formation of a fibrous stricture, depending on the vessel involved, the duration of the ischaemia and the bacterial flora. All these processes are well recognized by the pathologist. The conditions are seen much more frequently in the large than the small intestine (Marston et al, 1966, 1969; Marcuson, 1972), because of the design of its blood supply and the nature of its intraluminal flora. The causes and manifestations of this type of process form the subject of the discussion by Dr Morson.

\section{Summary and conclusion}

The splanchnic circulation contains between one fifth and one third of the blood volume at any one time, the proportion varying with exercise and with absorption. Anatomically, there are three zones supplied by the coeliac axis, and superior and inferior mesenteric arteries respectively, but because of the system of collateral supply, ischaemia becomes clinically important only in the middle of these, which involves the alimentary tract from jejunum to descending colon. Diminution of blood flow in the superior mesenteric artery, if continued below a certain critical point, brings about a cascade of physiological events which if uncorrected leads to death. The main components of this disturbance are haemoconcentration and absorption of vasoactive material from the ischaemic gut.

Gradual limitation of flow in the superior mesenteric artery as by an atheromatous stricture is usually well compensated by the opening up of a collateral circulation, so that detectable abnormalities are unusual, even if resting flow is reduced. Symptoms may be provoked by the failure of the mesenteric circulation to respond to ingestion of food, but these are difficult to correlate with studies of intestinal function. However, reduction of central arterial pressure from any cause may, in the presence of such an arterial block, lead to infarction.

Focal lesions in the small (or, more frequently the large) intestine, may occur from local vascular abnormalities. These vary from complete infarction to a transient inflammatory response.

\section{References}

Barclay, A. E., and Bentley, F. H. (1949). The vascularization of the human stomach: a preliminary note on the shuntim effect of trauma. British Journal of Radiology, 22, 62-67. ज)

Biber, B., Lundgren, O., and Svanvik, J. (1973). Intramural blood flow and blood volume in the small intestine of the cat as analysed by an indicator-dilution technique. $A c \mathbb{F}^{*}$ Physiologica Scandinavica, 87, 391-403.

Boley, S. J., Agrawal, G. P., Warren, A. R., Veith, F. Levowitz, B. S., Treiber, W., Dougherty, J., Schwartz, S. S., and Gliedman, M. L. (1969). Pathophysiologic effects \&f bowel distension on intestinal blood flow. Americ Journal of Surgery, 117, 228-234.

Boulter, P. S., and Parks, A. G. (1960). Submucosal vasculä patterns of the alimentary tract and their significancos British Journal of Surgery, 47, 546-550.

Burns, G. P., and Schenk, W. G., Jr. (1967). Intestinal blo숭 flow in the conscious dog. Surgical Forum, 18, 313-315. ⿷)

Burns, G. P., and Schenk, W. G., Jr. (1969). Effect of diges. tion and exercise on intestinal blood flow and cardiac output. Archives of Surgery, 98, 790-794.

Burton-Opitz, R. (1911). The vascularity of the liver. IV. T magnitude of the portal inflow. Quarterly Journal of $E_{\mho}$ perimental Physiology, 4, 113-125.

Chiu, C. J., Scott, H. J., and Gurd, F. N. (1972). Volume deficit versus toxic absorption-a study of canine shoct after mesenteric arterial occlusion. Annals of Surgery, 175, 479-488.

Dardik, H., Seidenberg, B., Parker, J. G., and Hurwitt, E. (1965). Intestinal angina with malabsorption treated by elective revascularization. Journal of the American Medical Association, 194, 1206-1210.

Delmont, J. P. (1971). In discussion, L'insuffisance arté dans les territoires du tronc coeliaque et des mésentériquiß supérieure et inférieure. Journal de Chirurgie (Paris), 10 .
213-236.

Kobold, E. E., and Thal, A. P. (1963). Quantitation ari identification of vasoactive substances liberated during various types of experimental and clinical intestinal ischemia. Surgery, Gynecology and Obstetrics, 117, 315-32ङ

Lefer, A. M. (1970). Role of a myocardial depressant factor the pathogenesis of circulatory shock. Federation Proceed ings, 29, 1836-1847.

Lundgren, O. (1967). Studies on blood flow distribution and countercurrent exchange in the small intestine. $A c \overline{\mathrm{g}}$ Physiologica Scandinavica, Suppl. 303.

Lundgren, $O$. (1974). The circulation of the small bow mucosa. Gut, 15, 1005-1013.

Marcuson, R. W. (1972). Ischaemic colitis. Clinics in Gastr\& enterology, 1, 745-763.

Marston, A. (1962). The bowel in shock. Lancet, 2, 365-378

Marston, A. (1963). Causes of death in mesenteric arteria occlusion. 1. Local and general effects of devascularizatiof of the bowel. 2. Observations on revascularization of the ischemic bowel. Annals of Surgery, 158, 952-969.

Marston, A. (1964). Patterns of intestinal ischaemia. Anna of the Royal College of Surgeons of England, 35, 151-181. Marston, A. (1977). Intestinal Ischaemia. Arnold, Londo周
(In Press).

Marston, A., Marcuson, R. W., Chapman, M., and Arthut J. F. (1969). Experimental study of devascularization of the colon. Gut, 10, 121-130.

Marston, A., Pheils, M. T., Thomas, M. L., and Morson B. C. (1966). Ischaemic colitis. Gut, 7, 1-10.

Morris, G. C., Jr., Crawford, E. S., Cooley, D. A., anto DeBakey, M. E. (1962). Revascularization of the celiac and superior mesenteric arteries. Archives of Surgery, 84, 95-10

Murrell, T. G. C., Roth, L., Egerton, J., Samels, J., an $\frac{\mathbb{P}}{\mathbb{R}}$ 
Walker, P. D. (1966). Pig-bel: Enteritis necroticans. Lancet, 1, 217-222.

Pawlik, W., and Jacobson, E. D. (1974). Effects of digoxin on the mesenteric circulation. Cardiovascular Research Center Bulletin, 12, 80-84.

Polansky, B. J., Berger, R. L., and Byrne, J. J. (1964). Massive non-occlusive intestinal infarction associated with digitalis toxicity. Circulation, 29/30, Suppl. 3, 141.

Reul, G. J., Jr., Wukasch, D. C., Sandiford, F. M., Chiarillo, L., Hallman, G. L., and Cooley, D. A. (1974). Surgery, 75, 682-689.

Saegesser, F., Phillips, V., Chapuis, G., and Rausis, C. (1972). Cancers associés à la rectocolite ulcéro-hémorrhagique et aux autres manifestations inflammatoires du gros intestin. Praxis, 61, 200-206.

Spanner, R. (1932). Neue Befunde über die Blutwege der Darmwand und ihre funktionelle Bedeutung. Morphologische Jahrbuch, 69, 394-454.

Svanvik, J. (1973). Mucosal hemodynamics in the small intestine of the cat during regional sympathetic vasocon- strictor activation. Acta Physiologica Scandinavica, 89, 1929.

Tjoing, B., Bella, E., Weiner, M., and Enquist, I. F. (1974). Fluid shifts and metabolic changes during and after occlusion of the superior mesenteric artery. Surgery, Gynecology and Obstetrics, 139, 217-221.

Vatner, S. F., Franklin, D., and van Citters, R. L. (1970). Mesenteric vasoactivity associated with eating and digestion in the conscious dog. American Journal of Physiology, 219, 170-174.

Wade, O. L., Combes, B., Childs, A. W., Wheeler, H. O., Cournand, A., and Bradley, S. E. (1956). The effect of exercise on the splanchnic blood-flow and splanchnic blood volume in normal man. Clinical Science, 15, 457-463.

Williams, L. F., Jr., Goldberg, A. H., Polansky, B. J., and Byrne, J. J. (1969). Myocardial effects of intestinal ischemia. Journal of Surgical Research, 9, 319-323.

Zeissler, J., and Rassfeld-Sternberg, L. (1949). Enteritis necroticans due to Clostridium welchii type F. British Medical Journal, 1, 267-269. 